\title{
The saturation state of strength and ductility of bimodal
}

\section{nanostructured metals}

\author{
X. Guo ${ }^{\mathrm{a}, *}$, G. Yang ${ }^{\mathrm{a}}$, G.J. Weng ${ }^{\mathrm{b}}$ \\ ${ }^{a}$ School of Mechanical Engineering, Tianjin University, Tianjin 300072, China \\ ${ }^{\mathrm{b}}$ Department of Mechanical and Aerospace Engineering, Rutgers University, New \\ Brunswick, NJ 08903, USA
}

Bimodal nanostructured metals have ultrahigh strength and good ductility, but their values are highly dependent on the cohesive strength of both phases. To explore this dependence, we apply the strain-gradient plasticity theory to the nanograined phase and a cohesive finite element method to determine the overall fracture process. The results show that when the cohesive strength of both phases reaches certain level, the stress-strain curves will reach a saturation state. This finding points to the significance of designing the cohesive strength to such a critical value so that the maximum strength and ductility could be attained.

Keywords: Bimodal nanostructured metals; Cohesive finite element method; Deformation and fracture; Microstructure; Saturation

\section{Introduction}

The bimodal nanostructured (NS) metals consist of two phases, i.e., the nanograined $(\mathrm{NG})$ and coarse-grained $(\mathrm{CG})$ phases. The NG region serves as the matrix and coarse-grains as the toughening inclusions. This class of materials has superb mechanical properties. The most significant are the high strength and good ductility [1-7]. In a recent numerical simulation, it was disclosed that these superb properties were attributed to crack bridging in CG inclusions and crack deflection in the NG matrix [8]. Another intriguing property is their superb ballistic performance [4]. More specifically they also have a higher limit velocity than the NG structure [9]. A series of experiments have been conducted to explore the underlying mechanisms. It has been generally concluded that crack blunting, crack bridging, debonding, and strain hardening in the $\mathrm{CG}$ inclusions are responsible for these superb properties [1,4,10-13]. But a more complete understanding remains a challenge. At present, research about the bimodal NS metals still continues.

* Corresponding author. E-mail: xiangguo@ @ tju.edu.cn (X. Guo). 
Our route of research is high-resolution computer simulations. To investigate the effects of microstructural attributes on the fracture behavior of $\mathrm{NS} \mathrm{Cu}$, the element deletion method has been widely used $[8,9]$. But it is unable to predict crack branching properly [14], and none of these investigations have considered the properties of interfaces. It turns out that the interface is a critical factor in both strength and ductility of bimodal metals. An excellent methodology to track the arbitrary nature of fracture processes at the meso-scale is by cohesive finite element method (CFEM). Within this framework, the intrinsic CFEM embeds the cohesive elements along all finite element boundaries and will be adopted. Recently we have also used it to investigate the layered stainless steel with NG interface layers and found that its ductility strongly depended on the brittleness and thickness of the NG layer [15-17]. The relations between microstructural parameters and fracture toughness can also be established [18]. As of now, CFEM investigations on the fracture process of bimodal NS metals are still very limited [19]. Our goal is to use the intrinsic CFEM to uncover how the cohesive strength of both NG and CG phases and the microstructural attributes of $\mathrm{CG}$ inclusions affect the overall strength and ductility of NS $\mathrm{Cu}$ under tensile loading.

\section{Method}

Four bimodal NS Cu microstructures $(60 * 300 \mu \mathrm{m})$ subjected to a tensile loading are chosen [8]. They, with the NG phase in green and the CG phase in red, possess the same volume fraction of the CG inclusions. Microstructure A (Fig. 1a1) consists of arrays of inclusions, while B (Fig. 1a2) has a staggered distribution. Microstructures AR (Fig. 1a3) and BR (Fig. 1a4) are obtained by replacing each circular inclusion in microstructures $\mathrm{A}$ and $\mathrm{B}$ with a square inclusion. The bilinear cohesive law is illustrated in Fig. $1 \mathrm{~b}$ with $T_{\max }$ cohesive strength and $G_{\text {coh }}$ fracture energy. Damage pertinent to the cohesive element can be defined [20]. Three types of cohesive elements exist. For brevity, cohesive elements within the CG (NG) phase are referred to as CG-CG (NG-NG) elements. Similarly, those in the interfaces of the two phases are referred to as CG-NG elements. The fracture energy is specified as follows: $630 \mathrm{~J} / \mathrm{m}^{2}$ for the CG-CG elements and $2230 \mathrm{~J} / \mathrm{m}^{2}$ for the NG-NG elements [21]. The ratio of the cohesive strength of the CG-CG elements to the yield strength of the CG phase is denoted $n_{\mathrm{CG}}$. Similarly, $n_{\mathrm{NG}}$ can be defined. The combination of $n_{\mathrm{CG}}$ and $n_{\mathrm{NG}}$ is denoted $n_{\mathrm{CG}}-n_{\mathrm{NG}}$. Both the fracture energy and the cohesive strength of CG-NG elements are taken as the average of those of the two phases. The microstructure 
contains 72000 triangular elements. A boundary velocity $1 \mathrm{~m} / \mathrm{s}$ is imposed at the top and bottom edges. Condition of plain strain is assumed to prevail.
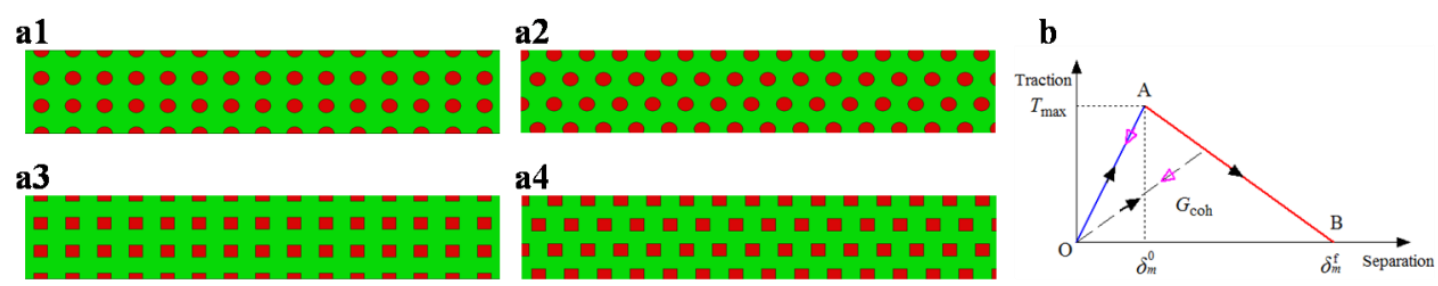

\section{Fig. 1}

The influence of grain boundaries in the NG phase needs to be considered [22]. We will fully account for their influence through consideration of geometrically necessary dislocations. This will be implemented by adopting the mechanism-based strain gradient plasticity theory to describe the constitutive relation of the NG phase [23]. The elastic strain rate is linear with the stress rate, while the plastic strain rate is proportional to the deviatoric stress. The equivalent plastic strain rate, $\dot{\varepsilon}_{\mathrm{e}}^{\mathrm{p}}$, can be determined as $\dot{\varepsilon}_{\mathrm{e}}^{\mathrm{p}}=\dot{\varepsilon}_{\mathrm{e}}\left[\frac{\sigma_{\mathrm{e}}}{\sigma_{\text {flow }}}\right]^{m_{0}}$ with $\dot{\varepsilon}_{\mathrm{e}}$ the equivalent strain rate, $\sigma_{\mathrm{e}}$ the von Mises stress, $m_{0}$ the strain rate-sensitivity parameter, and $\sigma_{\text {flow }}$ the flow stress of the NG phase [24]. The dislocation density in the grain boundary dislocation pile-up zones (GBDPZ), $\rho_{\mathrm{GB}}$, is used to consider the contribution of the grain boundaries. Taylor's model tells that $\sigma_{\text {flow }}=M \alpha \mu b \sqrt{\rho_{\mathrm{I}}+\rho_{\mathrm{GB}}}$ [25] where $M, \alpha, \mu$, and $b$ are the Taylor factor, the Taylor constant, the shear modulus, and the Burgers vector for the face-centered cubic $\mathrm{Cu}$, respectively, $\rho_{\mathrm{I}}$ is the dislocation density in the grain interior [26]. On the other hand, $\rho_{\mathrm{GB}}$ can be formulated by $\eta^{\mathrm{GB}}$ (the strain gradient in GBDPZ). By comparing the predictions of the above strain gradient plasticity theory with the experimental results of the NG phase, $\eta^{\mathrm{GB}}$ is fit to be $0.04 / \mathrm{nm}$ [23].

The Johnson-Cook plasticity model [27] considers effects of strain hardening, strain-rate hardening, and thermal softening. Using the calibrated strain gradient plasticity model, we can obtain the constitutive relation of the $\mathrm{NG} \mathrm{Cu}$ with $d_{\mathrm{G}} 23$ 
nm. The relation $\sigma_{\mathrm{e}}\left(\dot{\varepsilon}_{\mathrm{e}}^{\mathrm{p}}=\dot{\varepsilon}_{0}, T=T_{\mathrm{r}}\right)=A+B\left(\varepsilon_{\mathrm{e}}^{\mathrm{p}}\right)^{n} \quad\left(\dot{\varepsilon}_{0}\right.$ a reference strain rate, $T$ the temperature, $T_{\mathrm{r}}$ the room temperature) is used to fit the above constitutive relation.

The results of the NG Cu are $A=669 \mathrm{MPa}, B=912 \mathrm{MPa}$, and $n=0.28 \quad$ [8].

\section{Results and discussion}

Fig. 2 shows stress-strain curves of microstructure A. In Fig. $2 \mathrm{a}, n_{\mathrm{NG}}$ is fixed and $n_{\mathrm{CG}}$ is taken as $7,8,9$, and 10 in turn. When $n_{\mathrm{CG}}$ is 7 , the strength and ductility are relatively poor. When $n_{\mathrm{CG}}$ increases to 8,9 and 10 , both of them increase till stabilize. When $n_{\mathrm{NG}}$ increases to a higher level, with $n_{\mathrm{CG}}$ increasing, the relative increase in the strength and ductility decreases. Fig. 2 a indicates that $n_{\mathrm{NG}}=8$ is a critical value where both the strength and ductility are saturated. In Fig. $2 \mathrm{~b}, n_{\mathrm{CG}}$ is fixed and $n_{\mathrm{NG}}$ is taken as $3.5,4,4.5$, and 5 in turn. When $n_{\mathrm{NG}}$ is 3.5 , microstructure $\mathrm{A}$ has poor strength and ductility. Compared with the case of $n_{\mathrm{NG}}=3.5$, when $n_{\mathrm{NG}}$ increases to $4,4.5$, and 5 , its strength and ductility are improved, while the variation in the ductility is more prominent than that in the strength. The stress-strain curves corresponding to three $n_{\mathrm{CG}}-n_{\mathrm{NG}}$ almost coincide. When $n_{\mathrm{CG}}$ increases to a higher level, with $n_{\mathrm{NG}}$ increasing, the relative increase in the strength and ductility decreases. Fig. $2 b$ indicates that $n_{\mathrm{CG}}=4$ is another critical value where they are saturated. Therefore, the relationship $n_{\mathrm{CG}}-n_{\mathrm{NG}}$ corresponding to the saturation for microstructure $\mathrm{A}$ is $8-4$. This phenomenon also occurs in microstructures $\mathrm{B}, \mathrm{AR}$, and $\mathrm{BR}$. $n_{\mathrm{CG}} n_{\mathrm{NG}}$ corresponding to the saturation for B, AR, and BR are 8-4, 8-3.5, and 8-4, respectively.
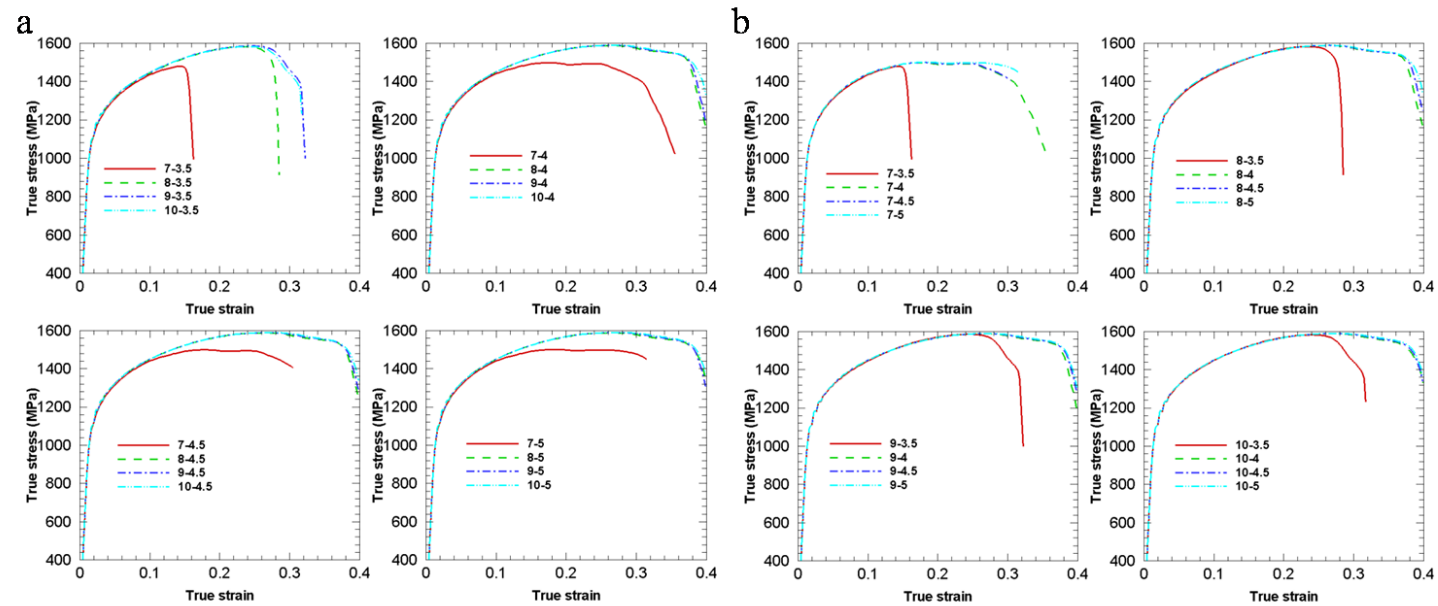

Fig. 2 
We analyze the saturation from a perspective of damage. For microstructure A, when $n_{\mathrm{NG}}=3.5$ and $n_{\mathrm{CG}}=7$, damage initiates in the $\mathrm{CG}$ inclusions. Subsequently, a microcrack initiates in the NG phase near the damaged CG inclusions. Two mode-I microcracks propagate from the left and right boundaries and then penetrate $\mathrm{CG}$ and NG phases successively, as illustrated in Fig. 3a. No severe plastic deformation (SPD) occurs in the other CG inclusions so that microcracks are not blocked effectively. When $n_{\mathrm{NG}}=3.5$ and $n_{\mathrm{CG}}=8$ or 9 , microcrack initiates in the NG phase. Since the cohesive strength of CG-CG elements increases, CG inclusions resist rapid propagation of the microcrack and play a bridging role. A mode-I microcrack penetrates the microstructure finally. When $n_{\mathrm{NG}}=3.5$ and $n_{\mathrm{CG}}=10$, a mode-I microcrack still initiates in the NG phase. In this case, SPD occurs in the CG inclusions, bridging works better, and then the CG inclusions in different rows bridge to bear the shear stress and mode-II microcracks initiate, as shown in Fig. 3b. Here, microcracks propagate in a mixed mode, which never occurs in the above cases.

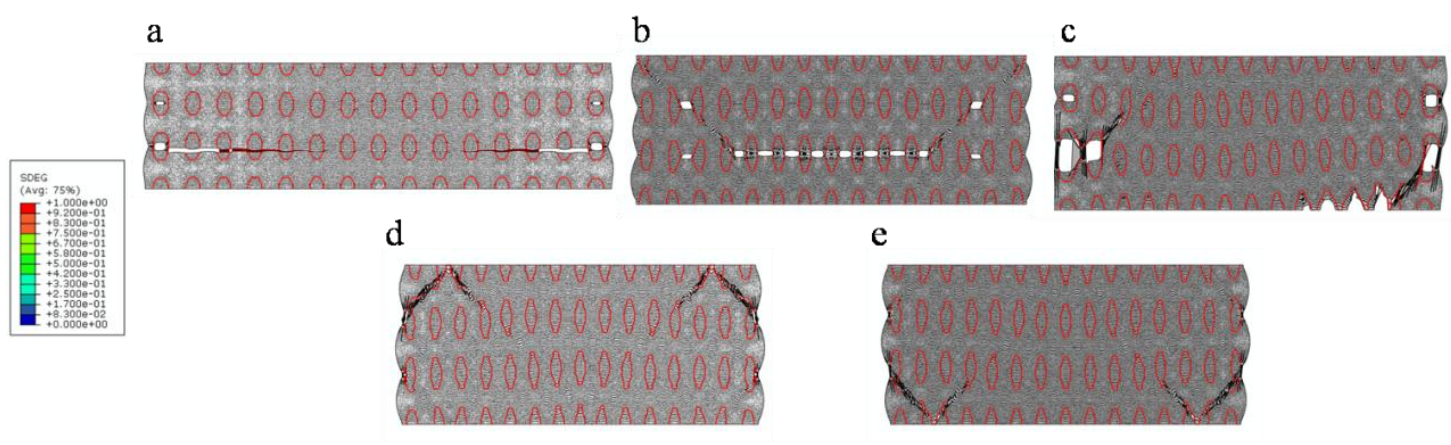

Fig. 3

When $n_{\mathrm{NG}}=4$ and $n_{\mathrm{CG}}=7$, some $\mathrm{CG}$ inclusions are damaged first. Its failure is more complicated than the case when $n_{\mathrm{NG}}=3.5$ and $n_{\mathrm{CG}}=7$, as illustrated in Fig. $3 \mathrm{c}$. Microcracks propagate continuously and form voids. The voids extend gradually. The NG region between different rows of $\mathrm{CG}$ inclusions is sheared and mode-II microcracks initiate. When $n_{\mathrm{NG}}=4$ and $n_{\mathrm{CG}}=8$, the NG phase is also damaged first and rapid propagation of the microcrack can be resisted, as shown in Fig. 3d. SPD occurs in some CG inclusions and bridging works, which blocks the mode-II microcracks effectively. The microcracks follow a zigzag path. When $n_{\mathrm{NG}}>4$ and $n_{\mathrm{CG}}>8$, the cohesive strength of CG-CG and NG-NG elements increases. However, we discover that microcrack initiation and propagation are similar with that in the case when $n_{\mathrm{NG}}=4$ and $n_{\mathrm{CG}}=8$. Fig. 3e illustrates the damage contours when $n_{\mathrm{NG}}=4$ and $n_{\mathrm{CG}}=9$. 
Generally, the above phenomenon can be summarized as follows. For microstructure A, when $n_{\mathrm{NG}} \leq 3.5$, cohesive strength of NG-NG elements is lower. With $n_{\mathrm{CG}}$ increasing, the location of microcrack initiation transits from CG phase to NG phase. Furthermore, with a low $n_{\mathrm{CG}}$, no SPD occurs in CG inclusions so that the capability to resist the propagation of microcrack deteriorates and bridging is not prominent. One or two mode-I microcracks occur and then penetrate microstructure A easily. When $n_{\mathrm{NG}} \geq 4$ and $n_{\mathrm{CG}} \leq 7$, main damage occurs in $\mathrm{CG}$ phase and then the damage occurs in NG phase as well. When $n_{\mathrm{NG}} \geq 4$ and $n_{\mathrm{CG}} \geq 8$, bridging is more prominent, mode-II microcracks follow zigzag paths, and the consistency of the failure patterns in microstructure A leads to the saturated stress-strain curves.

For microstructure B, failure patterns are similar with those for microstructure A. When $n_{\mathrm{NG}} \leq 3.5$, microcrack initiates mainly in the CG phase and the CG inclusions cannot effectively block the mode-I microcrack. When $n_{\mathrm{NG}} \geq 4$ and $n_{\mathrm{CG}}=7$, both the tensile damage of CG phase and the shear damage of NG phase dominate. However, when $n_{\mathrm{NG}} \geq 4$ and $n_{\mathrm{CG}} \geq 8$, the shear damage of the two phases dominates and bridging is prominent. For microstructure $\mathrm{AR}$, when $n_{\mathrm{NG}} \geq 3.5$ and $n_{\mathrm{CG}}=7$, the microcrack initiates in CG inclusions; when $n_{\mathrm{NG}} \geq 3.5$ and $n_{\mathrm{CG}} \geq 8$, it initiates in the NG phase. While in all cases, the NG phase is always sheared and the microcracks follow zigzag paths. For microstructure $\mathrm{BR}$, when $n_{\mathrm{NG}}=3.5$, mode-I microcracks penetrate two phases rapidly so its mechanical performance is worse; when $n_{\mathrm{NG}} \geq 4$ and $n_{\mathrm{CG}} \geq 8$, it is mainly sheared and mode-II microcracks dominate.

\section{Conclusions}

The influence of cohesive strength of each phase on the overall strength and ductility of bimodal NS Cu has been analyzed in this CFEM investigation. It has been found that when $n_{\mathrm{CG}}-n_{\mathrm{NG}}$ reaches certain level, both the strength and ductility will be saturated. Since the saturation state exists, the mechanical property appears to have an upper bound among the considered inclusion morphologies. As such, effort must be made to bring the cohesive strength of each phase to the critical level during the fabrication process.

Acknowledgments X.G. acknowledges the support from National NSF of China (Project nos. 11372214 and 11102128). G.J.W. thanks the support of NSF Program under CMMI-1162431. 


\section{References}

[1] Y. Wang, M. Chen, F. Zhou, E. Ma, Nature 419 (2002) 912-915.

[2] D.V. Witkin, Z. Lee, R. Rodriguez, S. Nutt, E.J. Lavernia, Scr. Mater. 49 (2003) 297-302.

[3] D.S. Gianola, S.V. Petegem, M. Legros, S. Brandstetter, H.V. Swygenhoven, K.J. Hemker, Acta Mater. 54 (2006) 2253-2263.

[4] A.P. Newbery, S.R. Nutt, E.J. Lavernia, JOM. 58(4) (2006) 56-61.

[5] D.K. Yang, P.D. Hodgson, C.E. Wen, Scr. Mater. 63 (2010) 941-944.

[6] A. Magee, L. Ladani, T.D. Topping, E.J. Lavernia, Acta Mater. 60 (2012) $5838-5849$.

[7] Z. Zhang, S.K. Vajpai, D. Orlov, K. Ameyama, Mater. Sci. Eng. A 598 (2014) $106-113$.

[8] X. Guo, R. Ji, G.J. Weng, L.L. Zhu, J. Lu, Mater. Sci. Eng. A 618 (2014) 479-489.

[9] X. Guo, G. Yang, G.J. Weng, L.L. Zhu, Mater. Sci. Eng. A 630 (2015) 13-26.

[10] B.Q. Han, J. Ye, F. Tang, J. Schoenung, E.J. Lavernia, J. Mater. Sci. 42 (2007) $1660-1672$.

[11] E.J. Lavernia, B.Q. Han, J.M. Schoenung, Mater. Sci. Eng. A 493 (2008) 207-214.

[12] O. Ertorer, T.D. Topping, Y. Li, W. Moss, E.J. Lavernia, Metall. Mater. Trans. A 42 (2011) 964-973.

[13] Q.H. Bui, J. Mater. Sci. 47 (2012) 1902-1909.

[14] J.H. Song, H. Wang, T. Belytschko, Comput. Mech. 42 (2008) 239-250.

[15] X. Guo, A.Y.T. Leung, A.Y. Chen, H.H. Ruan, J. Lu, Scr. Mater. 63 (2010) 403-406.

[16] X. Guo, G.J. Weng, A.K. Soh, Comput. Mater. Sci. 55 (2012) 350-355.

[17] X. Guo, W.J. Zhang, L.L. Zhu, J. Lu, Eng. Fract. Mech. 105 (2013) 211-220.

[18] X. Guo, K. Chang, L.Q. Chen, M. Zhou, Eng. Fract. Mech. 96 (2012) 401-415.

[19] A.C. Magee, L. Ladani, Mech. Mater. 82 (2015) 1-12.

[20] ABAQUS User's Manual, version 6.10. Dassault, Providence, RI (2013).

[21]A. Singh, L. Tang, M. Dao, L. Lu, S. Suresh, Acta Mater. 59 (2011) 2437-2446.

[22] G.J. Weng, Rev. Adv. Mater. Sci. 19 (2009) 41-62.

[23] L.L. Zhu, J. Lu, Int. J. Plast. 30-31 (2012) 166-184.

[24] Y. Huang, S. Qu, K.C. Hwang, M. Li, H.J. Gao, Int. J. Plast. 20 (2004) 753-782.

[25] H. Gao, Y. Huang, W.D. Nix, J.W. Hutchinson, J. Mech. Phys. Solids 47 (1999) 
1239-1263.

[26] U.F. Kocks, H. Mecking, Prog. Mater. Sci. 48 (2003) 171-273.

[27] G.R. Johnson, W.H. Cook, In: Proceedings of the 7th International Symposium on Ballistics, The Hague, The Netherlands (1983).

\section{Captions}

Fig. 1. Four idealized microstructures ((a1) microstructure A, (a2) microstructure B , (a3) microstructure AR, and (a4) microstructure BR) [14] and (b) bilinear cohesive law.

Fig. 2. Stress-strain curves of microstructure $\mathrm{A}$ when (a) $n_{\mathrm{NG}}$ is fixed and (b) $n_{\mathrm{CG}}$ is fixed.

Fig. 3. Damage contours in microstructure A when (a) $n_{\mathrm{CG}}-n_{\mathrm{NG}}=7-3.5$ and $t=5.4 \mu \mathrm{s}$, (b) $n_{\mathrm{CG}}-n_{\mathrm{NG}}=10-3.5$ and $t=11.9 \mu \mathrm{s}$, (c) $n_{\mathrm{CG}}-n_{\mathrm{NG}}=7-4$ and $t=13.4 \mu \mathrm{s}$, (d) $n_{\mathrm{CG}}-n_{\mathrm{NG}}=8-4$ and $t=15 \mu \mathrm{s}$, and (e) $n_{\mathrm{CG}}-n_{\mathrm{NG}}=9-4$ and $t=15 \mu \mathrm{s}$. Red marks between two phases are used to highlight phase boundaries and do not mean that the boundaries fail. 